\title{
Production of $\Lambda_{c}$ baryons at the LHC within the $k_{T}$-factorization approach and independent parton fragmentation picture
}

\author{
Rafał Maciuła $^{*}$ and Antoni Szczurek ${ }^{\dagger}$ \\ Institute of Nuclear Physics, Polish Academy of Sciences, \\ Radzikowskiego 152, PL-31-342 Kraków, Poland
}

(Received 5 April 2018; published 12 July 2018)

\begin{abstract}
We calculate the cross section for the production of $D$ mesons and $\Lambda_{c}$ baryons in proton-proton collisions at the LHC. The cross section for production of $c \bar{c}$ pairs is calculated within the $k_{T}$-factorization approach with the Kimber-Martin-Ryskin unintegrated gluon distributions obtained on the basis of modern collinear gluon distribution functions. We show that our approach well describes the $D^{0}, D^{+}$, and $D_{s}$ experimental data. We try to understand recent ALICE and LHCb data for $\Lambda_{c}$ production with the $c \rightarrow \Lambda_{c}$ independent parton fragmentation approach. The Peterson fragmentation functions are used. The $f_{c \rightarrow \Lambda_{c}}$ fragmentation fraction and $\varepsilon_{c}^{\Lambda}$ parameter for $c \rightarrow \Lambda_{c}$ are varied. As a control plot we show the transverse momentum distributions of different species of $D$ mesons assuming standard values of the $f_{c \rightarrow D}$ fragmentation fractions known from the literature. Although one can agree with the ALICE data using the standard estimation of model uncertainties, one cannot describe simultaneously the ALICE and the $\mathrm{LHCb}$ data with the same set of parameters. The fraction $f_{c \rightarrow \Lambda_{c}}$ necessary to describe the ALICE data is much larger than the average value obtained from $e^{+} e^{-}$or $e p$ experiments. No drastic modification of the shape of the fragmentation function is allowed by the new ALICE and LHCb data for $\Lambda_{c}$ production. We also discuss a possible dependence of the $\Lambda_{c} / D^{0}$ baryon-to-meson ratio on rapidity and transverse momentum as recent observations by the ALICE and LHCb collaborations seem to suggest. Three different effects are considered: the value of the $\varepsilon_{c}^{\Lambda}$ parameter in the Peterson fragmentation function for $c \rightarrow \Lambda_{c}$, a kinematical effect related to the hadronization prescription, and a possible feed-down from higher charmedbaryon excitations. It seems very difficult, if not impossible, to understand the ALICE data within the considered independent parton fragmentation scheme.
\end{abstract}

DOI: $10.1103 /$ PhysRevD.98.014016

\section{INTRODUCTION}

The production of charm ( $c \bar{c}$ pairs) belongs in principle to the domain of perturbative physics. The corresponding cross section can be calculated in the collinear factorization approach. The leading-order calculation is known to give a cross section that is too small, and thus the next-to-leadingorder (NLO) calculation must be performed (see, e.g. Refs., [1,2]). An effective and efficient alternative is the $k_{T}$-factorization approach [3-5]. The $k_{T}$ factorization provides a good description of $D$ meson production cross sections at the RHIC [6], Tevatron [7], and LHC [8,9].

*rafal.maciula@ifj.edu.pl

†Also at University of Rzeszów, PL-35-959 Rzeszów, Poland; antoni.szczurek@ifj.edu.pl

Published by the American Physical Society under the terms of the Creative Commons Attribution 4.0 International license. Further distribution of this work must maintain attribution to the author(s) and the published article's title, journal citation, and DOI. Funded by SCOAP ${ }^{3}$.
The production of $D$ mesons and/or nonphotonic leptons requires nonperturbative information about the hadronization process. To describe $D$-meson production fragmentation functions (FFs) for $c \rightarrow D$, quark-to-meson transitions are usually included. In the context of heavy-flavor production the Peterson FFs [10] are usually used. Other scale-independent fragmentation functions can also be found in the literature [11-13]. The effects on $D$-meson production related to the different FF models were discussed in Ref. [8]. Scale-dependent FFs for charm production that undergo evolution equations were proposed in Ref. [14]. The evolution equation also leads by construction to $g \rightarrow D$ fragmentation. Such an approach gives a good description of the LHC data at large transverse momenta $\left(p_{T}>2-3 \mathrm{GeV}\right)$ but overshoots experimental data at low transverse momenta $[9,15]$. However, the evolution approach was done only for massless quarks and it may be expected that the inclusion of a mass effect would probably change the results. Also, it is not clear how initial conditions for evolution should be included. A relatively large $g \rightarrow D$ transition leads to an unusually large $\sigma_{\text {eff }}$, a 
parameter for the double-parton scattering mechanism [16]. At large rapidities and low collision energies, subleading light quark/antiquark $q / \bar{q} \rightarrow D$ fragmentation may also be important [17].

Recently, the LHCb [18] and (very recently) ALICE [19] collaborations obtained new results for $\Lambda_{c}$ production at the highest collision energy so far, $\sqrt{s}=7 \mathrm{TeV}$. We wish to study whether the new LHCb and ALICE data can be described consistently within the chosen scheme of calculation based on $c \rightarrow \Lambda_{c}$ fragmentation. If so, it would be interesting to study whether the $f_{c \rightarrow \Lambda_{c}}$ fragmentation fraction is consistent with those found in previous studies of $e^{+} e^{-}, e p$, and $B$-meson decays.

\section{A SKETCH OF THE THEORETICAL FORMALISM}

\section{A. Parton-level calculations}

In the partonic part of our numerical calculations we follow the $k_{T}$-factorization approach. This approach is commonly known to be very efficient not only for inclusive particle distributions but also for studies of kinematical correlations. It was also shown many times by different authors that it provides very good description of heavy quark production in proton-proton collisions at different energies. Some time ago it was successfully used for theoretical studies of the $p p \rightarrow c \bar{c} X$ reaction at the LHC $[8,9]$. Very recently, this approach was also successfully applied, e.g., for $p p \rightarrow c \bar{c}+$ jet $X$ [20], $p p \rightarrow c \bar{c}+2$ jets $X$ [21], and $p p \rightarrow c \bar{c} c \bar{c} X$ [22].

According to this approach, the transverse momenta $k_{t}$ (virtualities) of both partons entering the hard process are taken into account and the sum of the transverse momenta of the final $c$ and $\bar{c}$ no longer cancels. Then the differential cross section at the tree level for the $c \bar{c}$-pair production reads

$$
\begin{aligned}
& \frac{d \sigma(p p \rightarrow c \bar{c} X)}{d y_{1} d y_{2} d^{2} p_{1, t} d^{2} p_{2, t}} \\
& =\int \frac{d^{2} k_{1, t}}{\pi} \frac{d^{2} k_{2, t}}{\pi} \frac{1}{16 \pi^{2}\left(x_{1} x_{2} s\right)^{2}} \overline{\left|\mathcal{M}_{g^{*} g^{*} \rightarrow c \bar{c}}^{\text {off }}\right|^{2}} \\
& \quad \times \delta^{2}\left(\vec{k}_{1, t}+\vec{k}_{2, t}-\vec{p}_{1, t}-\vec{p}_{2, t}\right) \mathcal{F}_{g}\left(x_{1}, k_{1, t}^{2}\right) \mathcal{F}_{g}\left(x_{2}, k_{2, t}^{2}\right),
\end{aligned}
$$

where $\mathcal{F}_{g}\left(x_{1}, k_{1, t}^{2}\right)$ and $\mathcal{F}_{g}\left(x_{2}, k_{2, t}^{2}\right)$ are the unintegrated gluon distribution functions (UGDFs) for both colliding hadrons and $\mathcal{M}_{g^{*} g^{*} \rightarrow c \bar{c}}^{\text {off-shell }}$ is the off-shell matrix element for the hard subprocess. The extra integration is over the transverse momenta of the initial partons. We keep exact kinematics from the very beginning and additional hard dynamics coming from the transverse momenta of incident partons. The explicit treatment of the transverse part of the momenta makes the approach very efficient in studies of correlation observables. The two-dimensional Dirac delta function assures momentum conservation. The unintegrated (transverse-momentum-dependent) gluon distributions must be evaluated at

$$
\begin{aligned}
& x_{1}=\frac{m_{1, t}}{\sqrt{s}} \exp \left(y_{1}\right)+\frac{m_{2, t}}{\sqrt{s}} \exp \left(y_{2}\right), \\
& x_{2}=\frac{m_{1, t}}{\sqrt{s}} \exp \left(-y_{1}\right)+\frac{m_{2, t}}{\sqrt{s}} \exp \left(-y_{2}\right),
\end{aligned}
$$

where $m_{i, t}=\sqrt{p_{i, t}^{2}+m_{c}^{2}}$ is the quark/antiquark transverse mass. In the case of charm-quark production at LHC energies, especially in the forward-rapidity region, one tests very small gluon longitudinal momentum fractions $x<10^{-5}$.

The matrix element squared for off-shell gluons is taken here in the analytic form proposed by Catani, Ciafaloni, and Hautmann $(\mathrm{CCH})$ [4]. It was also checked that the $\mathrm{CCH}$ expression is consistent with those presented later in Refs. [5,23] and in the limit of $k_{1, t}^{2} \rightarrow 0, k_{2, t}^{2} \rightarrow 0$ it converges to the on-shell formula.

The calculation of higher-order corrections in the $k_{t}$ factorization is much more complicated than in the case of the collinear approximation. However, the common statement is that in the $k_{t}$-factorization approach with tree-level off-shell matrix elements some part of the real higher-order corrections is effectively included. This is due to the possible emission of extra soft (and even hard) gluons encoded in the unintegrated gluon densities. More details of the theoretical formalism adopted here can be found in Ref. [8].

In the numerical calculation below we apply the KimberMartin-Ryskin (KMR) UGDF that is derived from a modified DGLAP-BFKL evolution equation [24,25] and which was found recently to work very well in the case of charm production at the LHC [8]. As also discussed in Ref. [20], the $k_{T}$-factorization approach with the KMR UGDF gives results that are well consistent with the collinear NLO approach. For the calculation of the KMR distribution we use here the up-to-date collinear MMHT2014 gluon parton distribution functions (PDFs) [26]. The renormalization and factorization scales $\mu^{2}=\mu_{R}^{2}=\mu_{F}^{2}=\frac{m_{1, t}^{2}+m_{2, t}^{2}}{2}$ and charm $=$ quark mass $m_{c}=1.5 \mathrm{GeV}$ are used in the present study. The uncertainties related to the choice of these parameters and to the collinear gluon PDFs will be discussed shortly when presenting numerical results.

\section{B. From quarks to hadrons}

The process of hadronization or parton fragmentation, i.e., the transition from partons to hadrons, has so far only been approached through phenomenological models. In principle, in the case of multiparticle final states the Lund string model [27] and the cluster fragmentation model [28] are often used. However, the fragmentation of heavy quarks 
in the independent parton model (in non-Monte-Carlo calculations) is usually done with the help of fragmentation functions. These objects provide the probability for finding a hadron produced from a high-energy quark or gluon.

According to the often-used formalism, the inclusive distributions of charmed hadrons $h=D, \Lambda_{c}$ are obtained through a convolution of inclusive distributions of charm quarks/antiquarks and $c \rightarrow h$ fragmentation functions:

$$
\left.\frac{d \sigma(p p \rightarrow h X)}{d y_{h} d^{2} p_{t, h}} \approx \int_{0}^{1} \frac{d z}{z^{2}} D_{c \rightarrow h}(z) \frac{d \sigma(p p \rightarrow c X)}{d y_{c} d^{2} p_{t, c}}\right|_{\substack{y=y_{h} \\ p t, c=p_{t, h} / z}},
$$

where $p_{t, c}=\frac{p_{t, h}}{z}$ and $z$ is the fraction of longitudinal momentum of a charm quark $c$ carried by a hadron $h=D, \Lambda_{c}$. A typical approximation in this formalism assumes that $y_{c}$ is unchanged in the fragmentation process, i.e., $y_{h}=y_{c}$. It was originally motivated for light hadrons but is also commonly accepted in the case of heavy hadrons.

As a default set in all of the following numerical calculations, the standard Peterson model of fragmentation function [10] with the parameters $\varepsilon_{c}^{D}=\varepsilon_{c}^{\Lambda}=0.05$ is applied. The parameter will be varied only in the case of the $c \rightarrow \Lambda_{c}$ transition. This choice of fragmentation function and parameters is based on our previous theoretical studies of open charm production at the LHC [8], where a detailed analysis of uncertainties related to the application of different models of FFs was done.

Another approach which makes use of phenomenological FFs is to assume that a hadron is emitted in the same direction as a charmed quark/antiquark, i.e., $\theta_{h}=\theta_{c}$, which is equivalent to $\eta_{h}=\eta_{c}$, where $\eta_{h}$ and $\eta_{c}$ are hadron and quark pseudorapidities. Here we follow the prescription presented in Ref. [29] where the fragmentation quantity $z$ was defined by the equation $E_{h}=z E_{c}$.

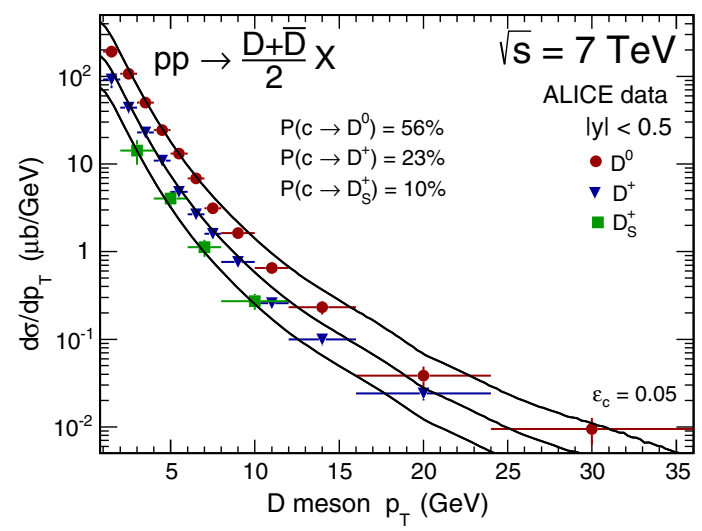

Finally, the calculated cross sections for the $D^{0}, D^{+}$, and $D_{S}^{+}$mesons and the $\Lambda_{c}$ baryon should be normalized to the relevant fragmentation fractions. For a nice review of the charm fragmentation fractions, see Ref. [30].

\section{RESULTS}

\section{A. Transverse momentum distributions of charmed mesons and baryons}

We start our presentation by showing results for $D$-meson production. In Fig. 1 we present transverse momentum distributions of different open charm mesons $D^{0}, D^{+}$, and $D_{s}$ for the ALICE (left panel) and the LHCb (right panel) kinematics. Here and throughout this subsection, the numerical results are obtained within the standard fragmentation procedure with the assumption of unchanged rapidity, i.e., $y_{c}=y_{h}$, where $h=D, \Lambda_{c}$. In this calculation we use the standard Peterson fragmentation function with $\varepsilon_{c}^{D}=0.05$ for the $c \rightarrow D$ transition. The fragmentation fractions for charmed mesons are set to be $f_{c \rightarrow D^{0}}=0.56$ and $f_{c \rightarrow D^{+}}=0.23$ for both the ALICE and $\mathrm{LHCb}$ detector acceptances. In the case of a charmedstrange meson two different values of the fragmentation fraction are needed to fit both data sets with the same precision, i.e., $f_{c \rightarrow D_{S}}=0.06$ for $\mathrm{LHCb}$ and 0.10 for ALICE. Both values of the fragmentation fraction for the $c \rightarrow D_{S}$ transition are consistent with those extracted from the combined analysis of charm-quark fragmentation fraction measurements in $e^{+} e^{-}, e p$, and $p p$ collisions [30]. We cannot describe both sets of data with the same $f_{c \rightarrow D_{s}}$. Doing so would yield a clear disagreement with, e.g., the $\chi^{2}$ criterion. It looks like there is a similar effect as for $\Lambda_{c}$, which we will discuss below.

Having fixed all parameters of the theoretical approach in the context of open charm meson production, we can proceed to the production of $\Lambda_{c}$ baryons. In Fig. 2 we present the transverse momentum distribution of $\Lambda_{c}$ baryons for the ALICE (left panel) and the LHCb (right panel)

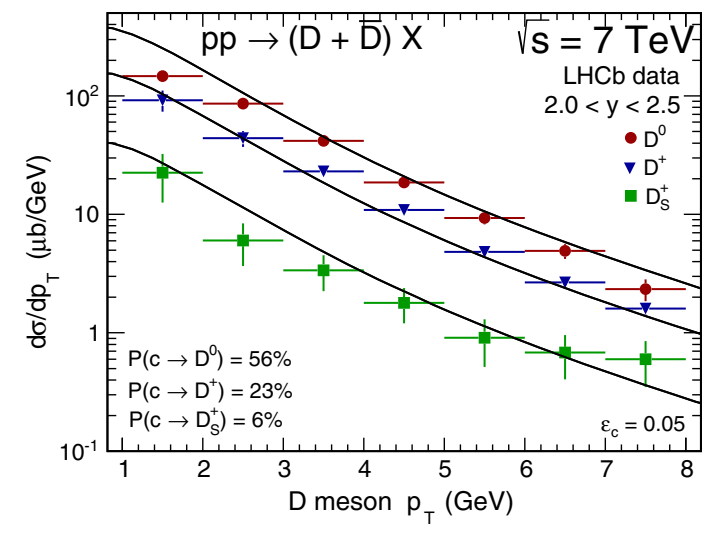

FIG. 1. Transverse momentum distribution of $D$ mesons for $\sqrt{s}=7 \mathrm{TeV}$ for ALICE (left panel) and LHCb (right panel). The experimental data points are taken from Refs. [31,18], respectively. 

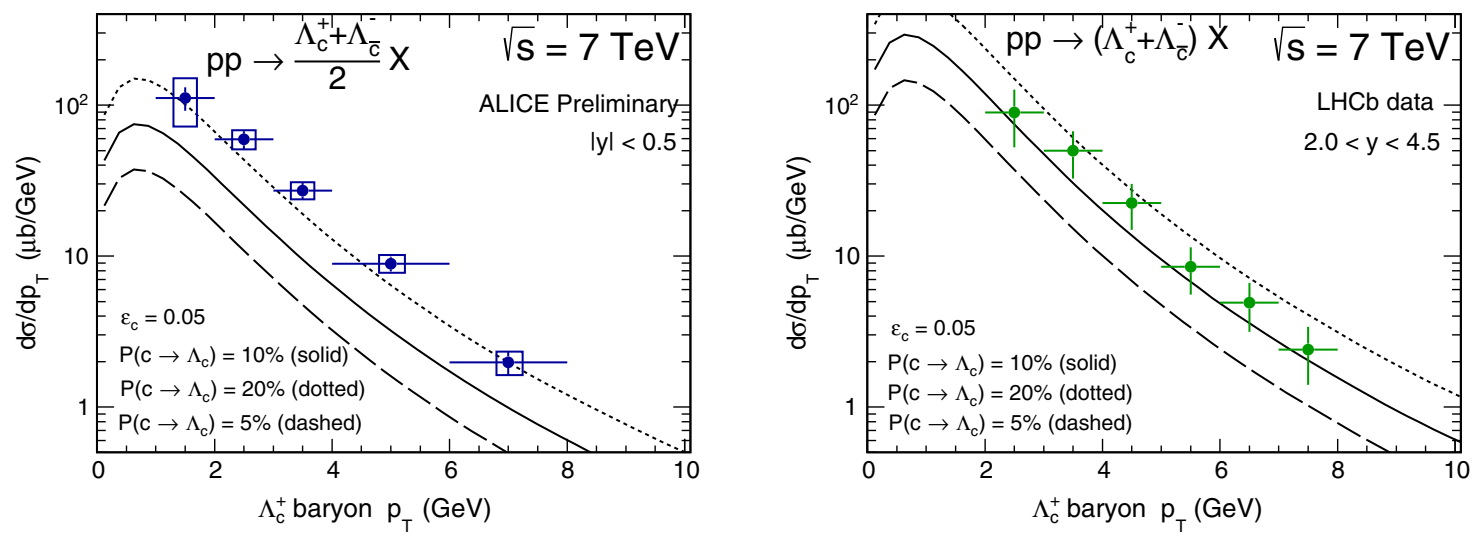

FIG. 2. Transverse momentum distribution of the $\Lambda_{c}$ baryon for $\sqrt{s}=7 \mathrm{TeV}$ for ALICE (left panel) and LHCb (right panel). The experimental data points are taken from Refs. [19,18], respectively.

kinematics. In this calculation we have also used the Peterson FF with the same parameter $\varepsilon_{c}^{\Lambda}=0.05$ (as a default) as for the $c \rightarrow D$ transition. The three lines correspond to different values of the $c \rightarrow \Lambda_{c}$ fragmentation fraction. The dashed curve is for $f_{c \rightarrow \Lambda_{c}}=0.05$, as is typical for pre-LHC results. Clearly, this result underpredicts both ALICE and LHCb data. We also show the results for increased fragmentation fractions, i.e., $f_{c \rightarrow \Lambda_{c}}=0.10$ (solid line) and 0.20 (dotted line). The agreement between data and the theoretical predictions with the increased $f_{c \rightarrow \Lambda_{c}}$ is better. However, a visible difference appears in the observed agreement for the midrapidity ALICE and forward LHCb regimes. Taking $f_{c \rightarrow \Lambda_{c}}=0.10$, we are able to describe the LHCb data quite well but we still underestimate the ALICE data by a factor $\sim 2$ in the whole considered range of transverse momenta. The shapes of the transverse momentum distributions are well reproduced in both the ALICE and LHCb cases. In order to get the right normalization in the case of the ALICE measurement, we need to take $f_{c \rightarrow \Lambda_{c}}=0.20$ which is much bigger than the numbers found in previous studies (see, e.g., the review in Ref. [30]).

The above results were obtained within the default set of calculation parameters: the charm-quark mass $m_{c}=1.5 \mathrm{GeV}$, the scales $\mu^{2}=\frac{m_{1, t}^{2}+m_{2, t}^{2}}{2}$, the KMR UGDF calculated from the MMHT2014lo gluon PDFs, and the running coupling $\alpha_{S}$, which is at the same order as the PDFs used here. In Fig. 3 we show, for completeness, the uncertainties related to the choice of the perturbative calculation parameters (left panel) and due to the choice of the gluon collinear PDFs in the calculation of the KMR UGDF (right panel). The upper limit of the somewhat arbitrary uncertainties almost agrees with the ALICE experimental data. However, this would not be the case for the LHCb data, where already the central value is consistent with the data (see right panel of Fig. 2). The set of parameters which describes the ALICE data for $\Lambda_{c}$ production would also lead to an overestimation of $D$-meson data.

Can the situation change when the shape of the $c \rightarrow \Lambda_{c}$ fragmentation function is different? As an illustration, in Fig. 4 we show results for different values of the $\varepsilon_{c}^{\Lambda}$
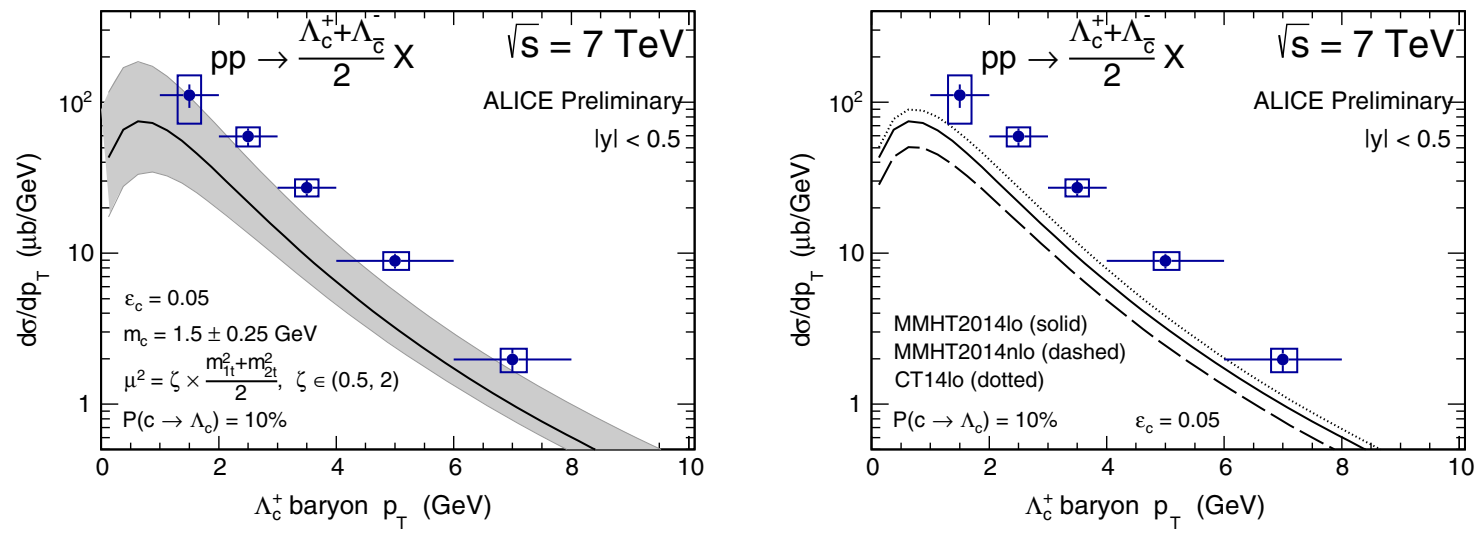

FIG. 3. Transverse momentum distribution of the $\Lambda_{c}$ baryon for $\sqrt{s}=7 \mathrm{TeV}$ for ALICE together with uncertainties related to the choice of the scales and the quark mass summed in quadrature (left) and due to the choice of the collinear gluon PDFs used in the calculation of the KMR UGDFs (right). Details are specified in the figure. The experimental data points are taken from Ref. [19]. 

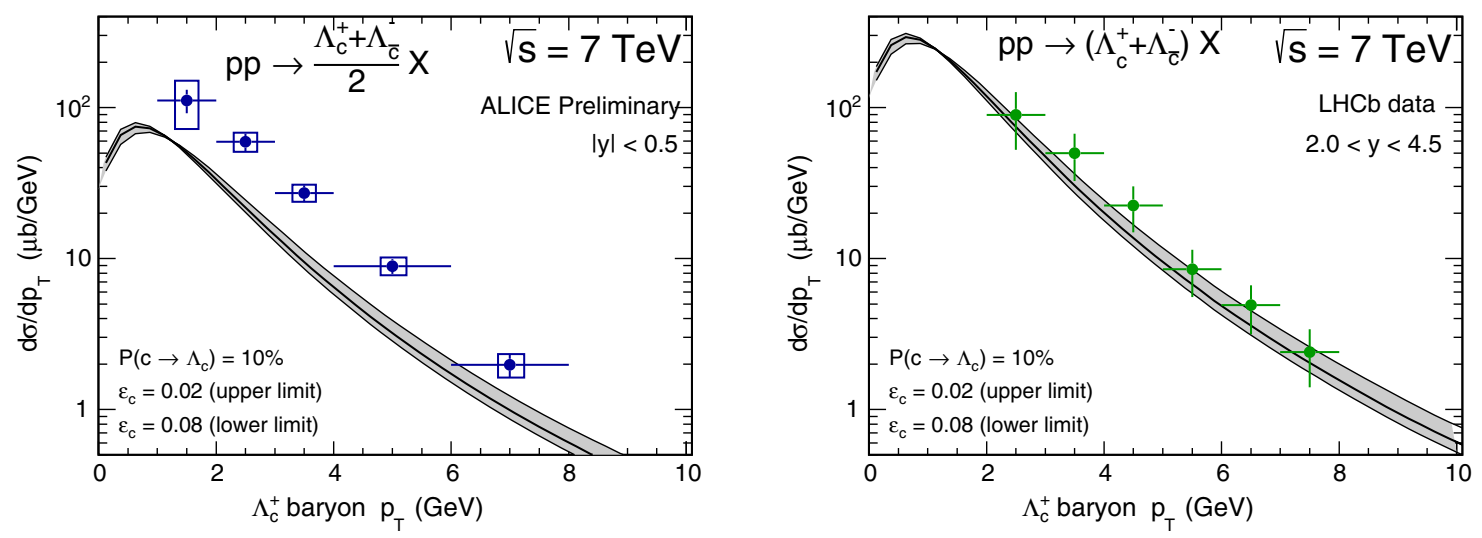

FIG. 4. Transverse momentum distribution of the $\Lambda_{c}$ baryon for $\sqrt{s}=7 \mathrm{TeV}$ for ALICE (left panel) and LHCb (right panel) for different values of the $\varepsilon_{c}^{\Lambda}$ parameter in the Peterson fragmentation function for the $c \rightarrow \Lambda_{c}$ transition.

parameter in the Peterson fragmentation function. As can be seen from the figure, a drastic modification of the parameter changes the shape of the distribution in a moderate way. Here, the LHCb data suggests a harder FF, i.e., a smaller $\varepsilon_{c}$ parameter than in the case of the $c \rightarrow D$ transition. Going to the region of very small transverse momenta $p_{T} \lesssim m_{c}$, the upper and lower limits start to reverse. However, it seems that playing with the shape of the fragmentation function will not help us understand the huge enhancement of $\Lambda_{c}$ production observed by the ALICE Collaboration. At the same time, we describe nicely the LHCb data with the same set of parameters.

\section{B. Possible reasons for the dependence of the $\Lambda_{c} / D^{0}$ ratio on transverse momentum and rapidity}

The ALICE Collaboration also reported a much larger $\Lambda_{c} / D^{0}$ baryon-to-meson ratio [19] than that measured by the LHCb Collaboration [18]. Is this a real effect? This observation seems to be consistent with the conclusions drawn above. Here we want to discuss possible reasons for the transverse-momentum dependence of the ratio.

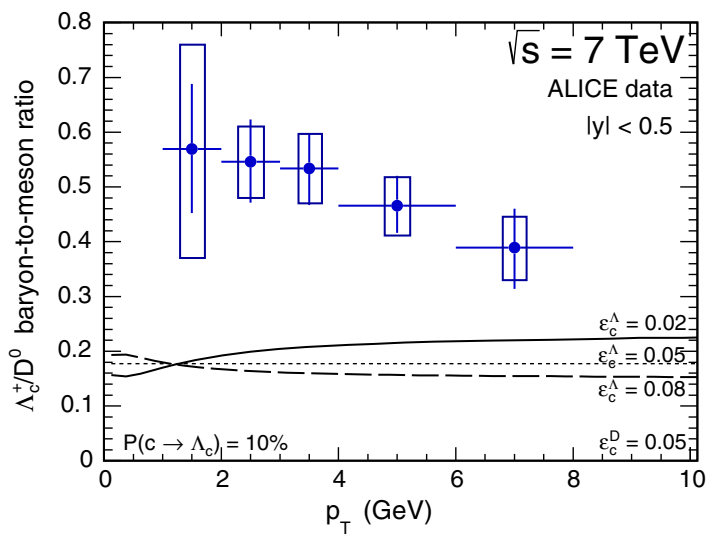

The fragmentation function for $c \rightarrow \Lambda_{c}$ does not need to be the same as for the $c \rightarrow D$ transition. This can be included even when using the Peterson FFs by choosing a different $\varepsilon_{c}$ parameter for fragmentation to a $D$ meson or a $\Lambda_{c}$ baryon. In Fig. 5 we show the ratio $\Lambda_{c} / D^{0}$ as a function of transverse momentum for ALICE (left panel) and LHCb (right panel). Here we keep $\varepsilon_{c}^{D}=0.05$ for the $c \rightarrow D$ fragmentation and take three different values $\varepsilon_{c}^{\Lambda}=0.02$ (solid line), 0.05 (dotted line), and 0.08 (dashed line) for the $c \rightarrow \Lambda_{c}$ transition. Again, by taking a smaller $\varepsilon_{c}$ parameter for the $c \rightarrow \Lambda_{c}$ case than for $c \rightarrow D$ fragmentation we are able to stay in agreement with the LHCb experimental data; however, we visibly underestimate the ALICE data. A similar situation was reported in Ref. [19] where the ALICE measurements were compared with results of different Monte Carlo event generators.

In the literature, the fragmentation of $c \rightarrow h$, where $h$ is a charmed meson or baryon, is usually done assuming that $y_{h}=y_{c}$. This is an approximation which was, in fact, not discussed carefully in the literature and which was originally derived for massless particles (both partons and hadrons). Here we wish to briefly discuss how the situation

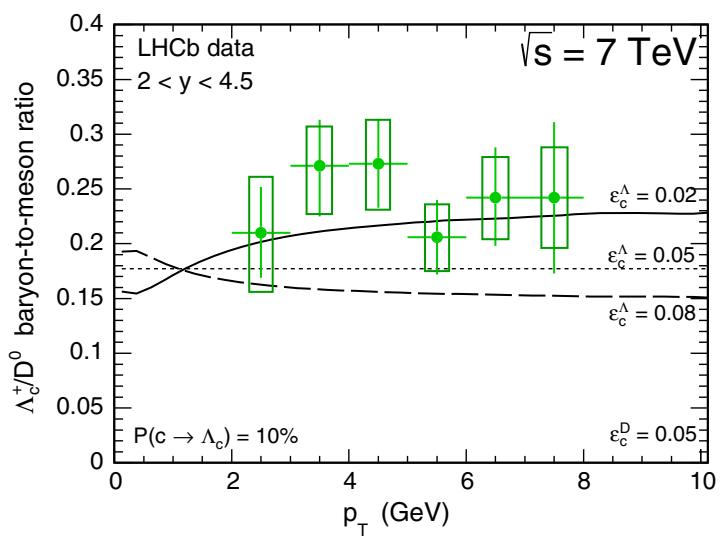

FIG. 5. Transverse momentum dependence of the $\Lambda_{c} / D^{0}$ baryon-to-meson ratio for ALICE (left) and LHCb (right) for different choices of the $\varepsilon_{c}^{\Lambda}$ parameter for the $c \rightarrow \Lambda_{c}$ transition in the Peterson fragmentation function. 


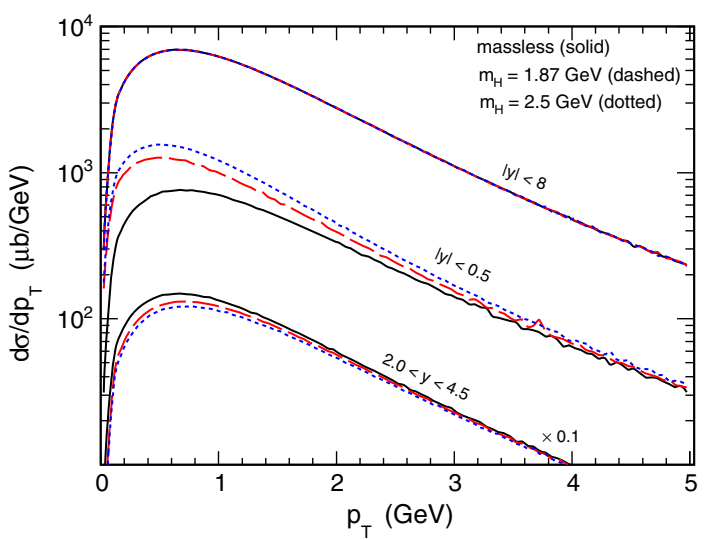

FIG. 6. Transverse momentum dependence of the cross section for different intervals of rapidities and for different approaches to the fragmentation procedure. The dotted and dashed lines correspond to the $\eta_{h}=\eta_{c}$ prescription for fragmentation. The solid lines are calculated with the standard $y_{h}=y_{c}$ approximation.

would change when a different fragmentation scheme is used. To illustrate the issue, we shall consider another approximation: $\eta_{h}=\eta_{c}$, i.e., we assume that the charmed hadron is emitted in the same direction (in the protonproton center-of-mass frame) as the quark/antiquark (see also Ref. [29]). Within this approach, the masses of the incident parton and final hadron are implicitly taken into account.

We try to compare the results of these two approximations in Fig. 6. The solid lines correspond to the standard $y_{h}=y_{c}$ approximation, while the dashed and dotted lines are calculated with the $\eta_{h}=\eta_{c}$ prescription, with $m_{h}=$ 1.87 and $2.5 \mathrm{GeV}$, respectively. Here we take $m_{h}=$ $2.5 \mathrm{GeV}$ which corresponds on average to the masses of $\Sigma_{c}$ baryons [32]. We observe that the latter approach leads to an enhancement of the cross section for small transverse momenta at midrapidities, which is the region relevant for the ALICE experiment. Simultaneously, an opposite effect is observed in the forward-rapidity region, where the cross section is slightly lowered with respect to the standard "massless" prescription.

Now we wish to show the size of the effect related to the new fragmentation scheme on the $\Lambda_{c} / D^{0}$ baryon-to-meson ratio. In Fig. 7 we observe only a small dependence of the ratio on the meson/baryon transverse momentum. However, comparing the left (ALICE) and right (LHCb) panels we observe slightly different values of the ratio. The change of the approximation leads to an enhancement of the ratio for the ALICE kinematics compared to the standard approach used in the literature. The enhancement is, however, only of the order of $10 \%$, but it is in the right direction. Here we only want to illustrate the effect of the enhanced production for ALICE related to the approximation made for fragmentation, so the $P\left(c \rightarrow \Lambda_{c}\right)$ is kept constant at the value known from other processes. However, the observed enhancement seems too small to explain the gigantic enhancement observed by the ALICE Collaboration.

So far only $\Lambda_{c}$ baryons have been measured in protonproton scattering. However, there is a multitude of predicted and/or observed singly charmed baryons: six with spin $1 / 2$ and six with spin $3 / 2$. We know nothing about their production in proton-proton collisions. Some of them could lead to a feed-down to a $\Lambda_{c}$ baryon [32]. Some of them decay weakly and could, in principle, be eliminated. Examples of interest are $\Sigma_{c}$ baryons both for $J=$ $1 / 2\left(\Sigma_{c}(2455)\right)$ and $J=3 / 2\left(\Sigma_{c}(2520)\right)$. The $\Sigma_{c}$ baryons are known to decay almost $100 \%$ of the time into $\Lambda_{c}$ and a pion [32].

So far we have implicitly assumed only the direct production of $\Lambda_{c}$ baryons (as represented by a Peterson fragmentation function with an a priori unknown $\varepsilon$ parameter). In principle, $\Lambda_{c}$ baryons do not need to be exclusively directly produced; they may come from a feeddown mechanism from excited baryonic states. The feeddown mechanism could modify transverse momentum distributions. The decay is done in a small Monte Carlo code. We assume isotropic decay in the rest frame of the

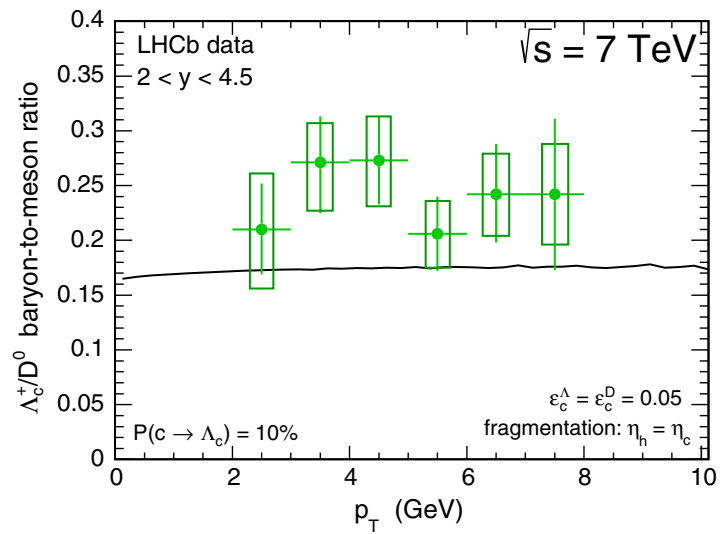

FIG. 7. Transverse momentum dependence of the $\Lambda_{c} / D^{0}$ baryon-to-meson ratio for ALICE (left) and LHCb (right) for the $\eta_{h}=\eta_{c}$ approximation. Here only one (default) set of $\varepsilon_{c}$ parameters for the Peterson fragmentation functions is used. 

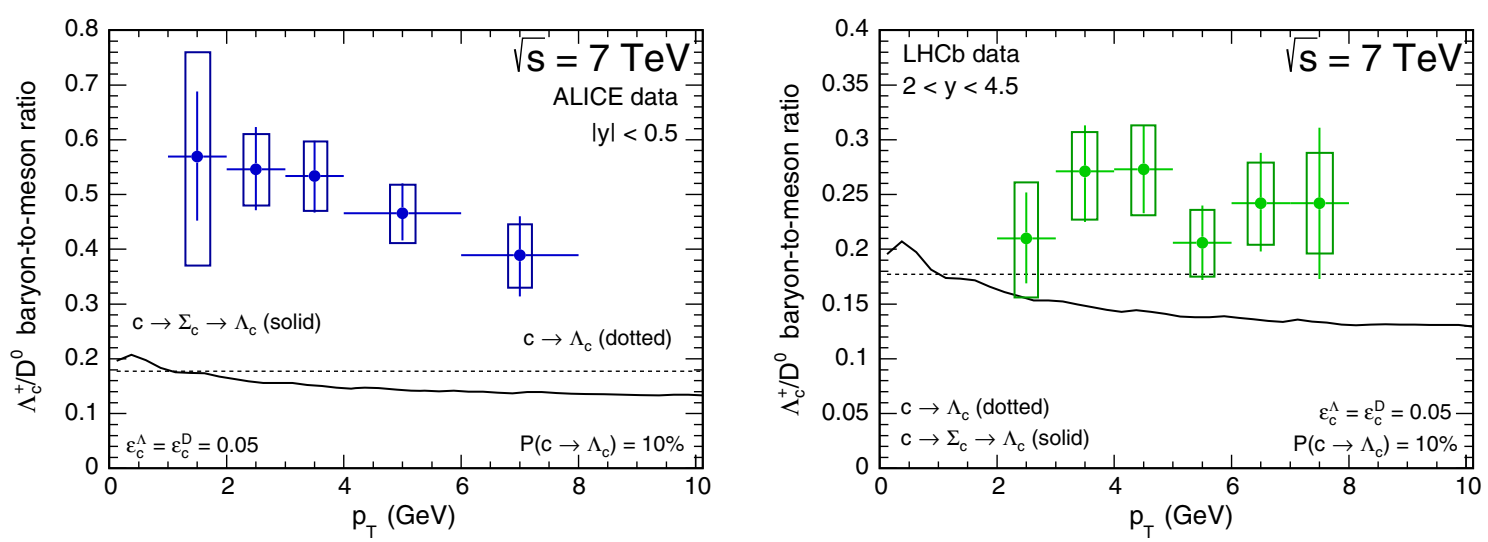

FIG. 8. Transverse momentum dependence of the $\Lambda_{c} / D^{0}$ baryon-to-meson ratio for ALICE (left) and LHCb (right) for the feed-down mechanism (solid lines). Here the standard $y_{h}=y_{c}$ fragmentation procedure with only one (default) set of $\varepsilon_{c}$ parameters for the Peterson fragmentation function was used. The dashed lines shown for reference correspond to the direct production $c \rightarrow \Lambda_{c}$.

excited state. Then, Lorentz boosts are performed to get the distribution in the laboratory (proton-proton center-ofmass) frame. In Fig. 8 we show the possible effect of such a feed-down. This mechanism leads to a small enhancement of the $\Lambda_{c} / D^{0}$ ratio at small transverse momenta, but it also causes it to decrease at larger $p_{T}$ 's. We conclude that the feed-down mechanism cannot explain the enhanced production observed by the ALICE Collaboration.

\section{CONCLUSIONS}

We have discussed the production of $\Lambda_{c}$ baryons in proton-proton collisions at LHC energies. The cross section for $c \bar{c}$ production has been calculated within the $k_{t}$-factorization approach. The Kimber-Martin-Ryskin unintegrated gluon distribution has been used. This combination ensures realistic charm rapidity and transverse momentum distributions. The hadronization has been performed within a simple independent parton fragmentation function formalism. The Peterson fragmentation function has been used in the present analysis. Varying the value of the $\epsilon_{c}$ parameter for the $c \rightarrow \Lambda_{c}$ transition modifies the shape of the transverse momentum distribution. A good agreement with the data (shape only) is obtained with a $\epsilon_{c}$ parameter of similar size as that found for $D$ mesons.

We found that the fragmentation fraction $f_{c \rightarrow \Lambda_{c}}=$ $0.1-0.15$ describes the recent data from the $\mathrm{LHCb}$ Collaboration but fails to describe the new ALICE data. Even for $\mathrm{LHCb}$, this number is slightly bigger than the values from the compilation of world results [30] obtained from experimental data on $e^{+} e^{-}, e p$, and $B$-meson decays. Although we could agree with the ALICE data using the standard estimation of model uncertainties related to the factorization/renormalization scale, quark mass, and PDF, we were not able to describe simultaneously the ALICE and LHCb $\Lambda_{c}$-baryon data as well as data on $D$-meson production with the same set of parameters.
The interpretation of the increased fragmentation fraction $c \rightarrow \Lambda_{c}$ is at present not clear and requires further studies, both on the theoretical and experimental sides.

We have also addressed the issue of the possible dependence of the $\Lambda_{c} / D^{0}$ ratio on rapidity and transverse momentum. Three different effects have been studied. We discussed how much the effect may depend on the not wellknown $\epsilon_{c}$ parameter in the Peterson fragmentation function for the $c \rightarrow \Lambda_{c}$ fragmentation. Only a small effect has been found. In addition, we have shown that a different treatment of the $c$ quark/antiquark fragmentation may slightly enhance the production of $\Lambda_{c}$ with respect to $D$ mesons at midrapidities. This effect is of purely kinematical origin and should not be visible in pseudorapidity distributions. Finally, we have discussed whether the indirect production of $\Lambda_{c}$ baryons could be related to the recent ALICE observation. For example, we considered a possible feed-down from $\Sigma_{c}$ baryons. A rather small effect of the shift down to small $p_{T}$ 's has been found. The effect for higher excitations (spin-3/2 $\Sigma_{c}$ baryons) is larger than for lower excitations (spin-1/2 $\Sigma_{c}$ baryons), which has a purely kinematical origin and is related to the masses of the $\Sigma_{c}$ baryons. A study of the production (and feed-down) of $\Sigma_{c}$ baryons will be possible with larger statistics from the ALICE Collaboration [33].

The independent parton fragmentation approach is only a simplification which has no firm and fundamental grounds, and thus requires tests to be considered as a valid approach. At low energies an asymmetry in the production of $\Lambda_{c}^{+}$and $\Lambda_{c}^{-}$was observed [34]. This may be related to the charmmeson cloud in the nucleon [35] and/or recombination with proton remnants [36]. At high energy this mechanism is active at large $x_{F}$ (or $\eta$, probably for pseudorapidities larger than those available for LHCb). Certainly, a study of $\Lambda_{c}^{+}-\Lambda_{c}^{-}$asymmetry in run II would be a valuable supplement. This would allow to verify the $c \rightarrow \Lambda_{c}$ "independent" parton hadronization picture. The new data from the 
ALICE Collaboration suggests a much bigger $f_{c \rightarrow \Lambda_{c}}$ hadronization fraction than those obtained in other processes and LHCb. In principle, it could even be the creation of $\Lambda_{c}$ in the quark-gluon plasma due to a coalescence mechanism (see, e.g., Ref. [37]). Such an enhancement was observed in $p-\mathrm{Pb}$ and $\mathrm{Pb}-\mathrm{Pb}$ collisions and interpreted in terms of the quark combination/coalescence approach in Refs. [38] (for $p$ - $\mathrm{Pb}$ ) and [39] (for Pb-Pb). Even in the "independent" parton picture, the hadronization fractions $f_{c \rightarrow D_{i}}$ and $f_{c \rightarrow \Lambda_{c}}$ do not need to be universal and may depend on their partonic surrounding associated with the collision which may be, in principle, reaction and energy dependent. Therefore, precise measurements at the LHC will allow to verify the picture and better understand the hadronization mechanism.
To experimentally explore the hypothesis that $\Lambda_{c}$ is produced in the mini quark-gluon plasma, one could study its production rates as a function of event multiplicity and compare them to similar analyses for the production of $D^{0}$ mesons.

\section{ACKNOWLEDGMENTS}

We are indebted to Alessandro Grelli and Andre Mishke for a discussion of the details of the ALICE experimental results for $\Lambda_{c}$ production in proton-proton collisions. This study was partially supported by the Polish National Science Center Grant No. DEC-2014/15/B/ST2/02528 and by the Center for Innovation and Transfer of Natural Sciences and Engineering Knowledge in Rzeszów.
[1] P. Nason, S. Dawson, and R. K. Ellis, Nucl. Phys. B327, 49 (1989); B335, 260(E) (1990).

[2] W. Beenakker, W. L. van Neerven, R. Meng, G. A. Schuler, and J. Smith, Nucl. Phys. B351, 507 (1991).

[3] S. Catani, M. Ciafaloni, and F. Hautmann, Phys. Lett. B 242, 97 (1990).

[4] S. Catani, M. Ciafaloni, and F. Hautmann, Nucl. Phys. B366, 135 (1991).

[5] J. C. Collins and R. K. Ellis, Nucl. Phys. B360, 3 (1991).

[6] R. Maciuła, A. Szczurek, and M. Łuszczak, Phys. Rev. D 92, 054006 (2015).

[7] H. Jung, M. Kraemer, A. V. Lipatov, and N. P. Zotov, J. High Energy Phys. 01 (2011) 085.

[8] R. Maciuła and A. Szczurek, Phys. Rev. D 87, 094022 (2013).

[9] A. Karpishkov, V. Saleev, and A. Shipilova, Phys. Rev. D 94, 114012 (2016).

[10] C. Peterson, D. Schlatter, I. Schmitt, and P. M. Zerwas, Phys. Rev. D 27, 105 (1983).

[11] V. G. Kartvelishvili, A. K. Likhoded, and V. A. Petrov, Phys. Lett. B 78, 615 (1978).

[12] P. D. B. Collins and T. P. Spiller, J. Phys. G 11, 1289 (1985).

[13] E. Braaten, K. m. Cheung, S. Fleming, and T. C. Yuan, Phys. Rev. D 51, 4819 (1995).

[14] T. Kneesch, B. A. Kniehl, G. Kramer, and I. Schienbein, Nucl. Phys. B799, 34 (2008).

[15] B. A. Kniehl, G. Kramer, I. Schienbein, and H. Spiesberger, Phys. Rev. D 79, 094009 (2009).

[16] R. Maciuła, V. A. Saleev, A. V. Shipilova, and A. Szczurek, Phys. Lett. B 758, 458 (2016).

[17] R. Maciuła and A. Szczurek, Phys. Rev. D 97, 074001 (2018).

[18] R. Aaij et al. (LHCb Collaboration), Nucl. Phys. B871, 1 (2013).

[19] S. Acharya et al. (ALICE Collaboration), J. High Energy Phys. 04 (2018) 108.
[20] R. Maciuła and A. Szczurek, Phys. Rev. D 94, 114037 (2016).

[21] R. Maciuła and A. Szczurek, Phys. Rev. D 96, 074013 (2017).

[22] R. Maciuła and A. Szczurek, Phys. Rev. D 87, 074039 (2013).

[23] R. Ball and R. K. Ellis, J. High Energy Phys. 05 (2001) 053.

[24] M. A. Kimber, A. D. Martin, and M. G. Ryskin, Phys. Rev. D 63, 114027 (2001).

[25] G. Watt, A. D. Martin, and M. G. Ryskin, Eur. Phys. J. C 31, 73 (2003).

[26] L. A. Harland-Lang, A. D. Martin, P. Motylinski, and R. S. Thorne, Eur. Phys. J. C 75, 204 (2015).

[27] B. Andersson, G. Gustafson, G. Ingelman, and T. Sjostrand, Phys. Rep. 97 (1983) 31.

[28] B. Webber, Nucl. Phys. B238, 492 (1984).

[29] M. Czech and A. Szczurek, Phys. Rev. C 72, 015202 (2005).

[30] M. Lisovyi, A. Verbytskyi, and O. Zenaiev, Eur. Phys. J. C 76, 397 (2016).

[31] S. Acharya et al. (ALICE Collaboration), Eur. Phys. J. C 77, 550 (2017).

[32] C. Patrignani et al. (Particle Data Group), Chin. Phys. C 40, 100001 (2016).

[33] A. Grelli (private communication).

[34] F. G. Garcia et al. (SELEX Collaboration), Phys. Lett. B 528, 49 (2002).

[35] E. R. Cazaroto, V. P. Goncalves, F. S. Navarra, and M. Nielsen, Phys. Lett. B 724, 108 (2013).

[36] C. Avila, J. Magnin, and L. M. Mendoza-Navas, arXiv:hep$\mathrm{ph} / 0307358$.

[37] J. Song, H. h. Li, and F. 1. Shao, Eur. Phys. J. C 78, 344 (2018).

[38] H. H. Li, F. L. Shao, J. Song, and R. Q. Wang, Phys. Rev. C 97, 064915 (2018).

[39] S. Plumari, V. Minissale, S. K. Das, and V. Greco, Eur. Phys. J. C 78, 348 (2018). 